\title{
Autonomic dysfunction following COVID-19 infection: an early experience
}

\author{
Kamal Shouman ${ }^{1}$. Greg Vanichkachorn ${ }^{2}$. William P. Cheshire ${ }^{3} \cdot$ Mariana D. Suarez $^{1}$ - Shahar Shelly ${ }^{1}$. \\ Guillaume J. Lamotte ${ }^{1}$. Paola Sandroni ${ }^{1}$. Eduardo E. Benarroch ${ }^{1}$. Sarah E. Berini ${ }^{1}$ - Jeremy K. Cutsforth-Gregory ${ }^{1}$. \\ Elizabeth A. Coon ${ }^{1} \cdot$ Michelle L. Mauermann ${ }^{1}$. Phillip A. Low ${ }^{1} \cdot$ Wolfgang Singer $^{1}$ (i)
}

Received: 2 March 2021 / Accepted: 25 March 2021 / Published online: 16 April 2021

(c) Springer-Verlag GmbH Germany, part of Springer Nature 2021

\begin{abstract}
Purpose Post-COVID-19 syndrome is a poorly understood aspect of the current pandemic, with clinical features that overlap with symptoms of autonomic/small fiber dysfunction. An early systematic analysis of autonomic dysfunction following COVID-19 is lacking and may provide initial insights into the spectrum of this condition.

Methods We conducted a retrospective review of all patients with confirmed history of COVID-19 infection referred for autonomic testing for symptoms concerning for para-/postinfectious autonomic dysfunction at Mayo Clinic Rochester or Jacksonville between March 2020 and January 2021.

Results We identified 27 patients fulfilling the search criteria. Symptoms developed between 0 and 122 days following the acute infection and included lightheadedness (93\%), orthostatic headache (22\%), syncope (11\%), hyperhidrosis (11\%), and burning pain (11\%). Sudomotor function was abnormal in 36\%, cardiovagal function in $27 \%$, and cardiovascular adrenergic function in $7 \%$. The most common clinical scenario was orthostatic symptoms without tachycardia or hypotension (41\%); $22 \%$ of patients fulfilled the criteria for postural tachycardia syndrome (POTS), and 11\% had borderline findings to support orthostatic intolerance. One patient each was diagnosed with autoimmune autonomic ganglionopathy, inappropriate sinus tachycardia, vasodepressor syncope, cough/vasovagal syncope, exacerbation of preexisting orthostatic hypotension, exacerbation of sensory and autonomic neuropathy, and exacerbation of small fiber neuropathy.

Conclusion Abnormalities on autonomic testing were seen in the majority of patients but were mild in most cases. The most common finding was orthostatic intolerance, often without objective hemodynamic abnormalities on testing. Unmasking/ exacerbation of preexisting conditions was seen. The temporal association between infection and autonomic symptoms implies a causal relationship, which however cannot be proven by this study.
\end{abstract}

Keywords Autonomic diseases · Postural orthostatic tachycardia syndrome $\cdot$ Autoimmune autonomic ganglionopathy Orthostatic intolerance $\cdot$ COVID-19

Wolfgang Singer

singer.wolfgang@mayo.edu

1 Department of Neurology, Mayo Clinic, 200 First Street SW, Rochester, MN 55905, USA

2 Preventive, Occupational, and Aerospace Medicine, Mayo Clinic, 200 First Street SW, Rochester, MN 55905, USA

3 Department of Neurology, Mayo Clinic, 4500 San Pablo Rd, Jacksonville, FL 32224, USA

\section{Introduction}

The novel severe acute respiratory syndrome coronavirus type 2 (SARS-CoV-2) and resulting coronavirus disease 2019 (COVID-19) emerged in late 2019 and quickly evolved into a pandemic that posed unprecedented challenges to the global health care system. As of the writing of this document, there have been 28,285,544 COVID-19 cases and 508,949 deaths reported in the United States alone [1]. Not only has SARS-CoV-2 infection been associated with multiple para-infectious neurological and autonomic abnormalities [2, 3], but postinfectious continuation or emergence of such derangements has also plagued many of the 
affected patients, a phenomenon known as "long COVID" [4], referred to as post-COVID syndrome at Mayo Clinic. The latter has attracted much interest due to the significant functional limitations experienced by sufferers and has garnered the attention of providers interested in autonomic medicine due to the associated symptoms of orthostatic and exercise intolerance. Case reports have recently been published of postural tachycardia syndrome (POTS) as a postinfectious complication of SARS-CoV-2 $[5,6]$. Others have reported post-COVID-19 POTS associated with other signs of autonomic dysfunction such as hyperhidrosis [7]. Cases of autonomic dysfunction beyond POTS have also been reported, including phenomena such as small fiber neuropathy with orthostatic cerebral hypoperfusion syndrome [8] and post-COVID-19 exacerbation of paroxysmal hypothermia and hyperhidrosis [9]. Post-COVID-19 acute inflammatory demyelinating polyneuropathy (AIDP), known for its potential association with autonomic dysfunction, has been described as well [10,11].

All of this highlights the need for a more systematic attempt at capturing the bigger picture of autonomic dysfunction in relation to COVID-19 via the investigation of a larger cohort of patients, utilizing careful neurological assessment and standardized autonomic function testing.

Our goal here is to share the experience of Mayo Clinic's large autonomic laboratories, with combined testing volumes of over 7000 patients a year, in order to provide a more comprehensive and less biased perspective regarding the autonomic impairment that post-COVID patients experience, in hopes that this report will lead to better understanding and management of autonomic dysfunction following COVID-19 infection, and help guide future prospective studies.

\section{Methods}

\section{Patients}

This is a retrospective analysis undertaken with the approval of the Mayo Clinic Institutional Review Board (IRB). Using an electronic search of the Mayo Clinic medical record system, we identified all patients who were referred for autonomic function testing at either Mayo Clinic Rochester, MN, or Jacksonville, FL, between March 2020 and January 2021 for symptoms concerning for autonomic dysfunction that emerged or were exacerbated after a laboratory-confirmed (polymerase chain reaction [PCR] and/or antibody testing) SARS-CoV-2 infection. The search results were expanded with a few additional cases encountered by the authors in the autonomic laboratory or autonomic clinic that were not captured in the original search. Patients were excluded if they had a suspected but not laboratory-confirmed infection.

\section{Clinical variables}

We then conducted a detailed medical record review of identified cases to derive demographic information (age, sex), COVID-19 infection details (date, symptoms, date of confirmatory testing), symptoms compatible with autonomic dysfunction (type, onset, duration), and findings on neurological examination. After review of all available clinical information and results of autonomic function testing, the most appropriate autonomic diagnosis was determined $[12,13]$.

\section{Autonomic function testing}

All patients underwent standardized autonomic function testing [14] with autonomic reflex screen (ARS) and/or thermoregulatory sweat test (TST). The ARS assesses postganglionic sympathetic sudomotor, cardiovagal, and cardiovascular adrenergic function. Postganglionic sympathetic sudomotor function is assessed using quantitative sudomotor axon reflex testing (QSART) [15], cardiovagal function by quantifying heart rate responses to deep breathing and heart rate responses to the Valsalva maneuver [16], and cardiovascular adrenergic function by assessing blood pressure responses to the Valsalva maneuver and passive head-up tilt [17]. All indices were compared to normative values for age and sex. A composite autonomic severity score (CASS) was derived from the ARS, which allows for quantification of severity and distribution of autonomic failure [18]. CASS is a validated instrument and is composed of three subdomains: sudomotor (score range $0-3)$, cardiovagal $(0-3)$, and adrenergic $(0-4)$. Total CASS ranges from 0 to 10 , with scores from 7 to 10 indicating severe autonomic failure.

The TST investigates sudomotor pathways from the hypothalamus to the eccrine sweat gland by quantifying areas of anhidrosis across the entire anterior body surface [19]. It is a standardized clinical procedure performed in a temperatureand humidity-controlled environment. The body core temperature is raised to $38{ }^{\circ} \mathrm{C}$ by standardized elevation of the environmental temperature while the patient is lying supine. The skin is covered with an indicator powder that changes color when exposed to sweat. Quantification of the percentage of anhidrosis is performed using digital photography.

\section{Statistics}

Summary statistics were used to describe demographic, clinical, and autonomic variables including median, range, frequencies, and percentages. 


\section{Results}

We identified 27 patients who met the criteria for confirmed COVID-19 diagnosis and autonomic symptoms that developed at or after the infection and continued, leading to referral for autonomic nervous system testing.

\section{Patient characteristics}

Patient characteristics are detailed in Table 1. Sixteen patients $(59 \%)$ were female and 11 were male (41\%). Ages ranged from 21 to 77 years, with a median of 30 years. Time from onset of first COVID-19 symptoms to COVID-19 diagnosis ranged from 0 to 16 days (median: 0 ), time from onset of first COVID-19 symptoms to autonomic symptoms ranged from 0 to 122 days (median: 7), and time from onset of first COVID-19 symptoms to autonomic testing ranged from 25 to 267 days (median: 119).

Eleven (41\%) patients developed autonomic symptoms during the infection and 16 (59\%) after infection. Symptoms compatible with autonomic/small fiber dysfunction included lightheadedness (93\%), orthostatic headache (22\%), syncope (11\%), hyperhidrosis (11\%), burning pain (11\%), orthostatic tachycardia (7\%), flushing (7\%), and weight loss (7\%). Hypohidrosis, heat intolerance, sicca syndrome, urinary hesitancy, urinary urgency, early satiety, blurry vision, numbness, and allodynia were each reported by one patient only.

\section{Autonomic testing results}

Details of autonomic function testing are summarized in Table 2. Autonomic function testing was abnormal in 17/27 $(63 \%)$ patients. CASS ranged from 0 to 8 , with a median of $1(n=22$; five cases were excluded due to possible anticholinergic medication effects). Sudomotor $(n=22)$, cardiovagal $(n=22)$, and cardiovascular adrenergic $(n=27)$ subscores were as follows (range, median): $(0-3,0),(0-3,0)$, and $(0-4$, 0 ), respectively. Quantitative sudomotor axon reflex test was abnormal in $8 / 22(36 \%)$, cardiovagal testing was abnormal in $6 / 22(27 \%)$, and cardiovascular adrenergic function was abnormal in $2 / 27$ (7\%) patients.

Maximum systolic blood pressure change on tilt ranged from -54 to $+18 \mathrm{mmHg}$ (median: -8 ), and maximum diastolic blood pressure change ranged from -20 to $+25 \mathrm{mmHg}$ (median: 0), respectively. Maximum heart rate increase on tilt ranged from 5 to 66 beats per minute (median: 23). Values are one-minute averages.

Symptoms were reported during head-up tilt by $22 / 27$ $(81 \%)$ patients. Lightheadedness was reported by $17(63 \%)$, headache by seven $(26 \%)$, tremulousness by four $(15 \%)$, warmth by four (15\%), shortness of breath by seven (26\%), shoulder pain by one (4\%), palpitations by two (7\%), hyperhidrosis by three (11\%), chest pain by five (19\%), leg heaviness by three (11\%), foot numbness by one (4\%), tinnitus by one (4\%), and abdominal pain by one (4\%).

Four patients had a thermoregulatory sweat test; all were abnormal. Two had widespread anhidrosis $(73.2 \%$ and $78.2 \%$ ) and two had a length-dependent pattern of sweat loss (4\% and $14.1 \%)$.

\section{Diagnosis}

The most commonly encountered clinical scenario was symptoms of orthostatic intolerance without demonstrable orthostatic tachycardia or orthostatic hypotension $(n=11$, $41 \%$ ); these patients would best be categorized as having subjective orthostatic intolerance (OI). Six patients (22\%) fulfilled diagnostic criteria for POTS [20], and an additional three patients (11\%) had orthostatic symptoms along with borderline tilt findings (borderline heart rate increase; borderline blood pressure drop), consistent with mild orthostatic intolerance. The following diagnoses were made in one patient each: autoimmune autonomic ganglionopathy (AAG), inappropriate sinus tachycardia, vasodepressor syncope, cough/vasovagal syncope, exacerbation of sensory predominant autonomic neuropathy, exacerbation of small fiber neuropathy, and exacerbation of neurogenic orthostatic hypotension/alpha-synucleinopathy.

\section{Representative cases}

\section{Postural tachycardia syndrome}

Patient 15 A 35-year-old woman developed cervical lymphadenopathy, diarrhea, and nausea. A SARS-CoV-2 test by nasal swab PCR 10 days after onset of symptoms was positive. Two months later, she started experiencing constant tingling in the feet, orthostatic lightheadedness, and brain fog. Over the next month, her symptoms continued to worsen despite a rehabilitative physical therapy program. Activities of daily living became impaired by orthostatic intolerance progressing to presyncope and numbness and burning pain in the lower legs with associated allodynia. She was evaluated by a neurologist, and electromyography (EMG) and magnetic resonance imaging (MRI) of the cervical and thoracic spine were normal. Treatment with tramadol, bupropion, and prednisone was unhelpful.

She presented to us with persistent symptoms 2 months after onset. Neurological examination revealed mild distal sensory loss to pinprick and temperature and was otherwise normal. ARS and TST showed symptomatic orthostatic tachycardia on tilt (heart rate increase of 31 beats per minute $[\mathrm{bpm}]$ associated with feelings of lightheadedness and 
Table 1 Patient characteristics

\begin{tabular}{|c|c|c|c|c|c|c|}
\hline $\mathrm{Pt}$ & Sex & Age & $\begin{array}{l}\text { COVID-19 Sx } \\
\text { to autonomic Sx } \\
\text { (days) }\end{array}$ & COVID-19 Sx & Autonomic presenting $\mathrm{Sx}$ & Autonomic Dx \\
\hline 1 & $\mathrm{~F}$ & 35 & 122 & $\begin{array}{l}\text { Cough, fever, LH myalgia, sore } \\
\text { throat, anosmia, dysgeusia, } \\
\text { congestion, rhinorrhea }\end{array}$ & LH, hyperhidrosis & $\begin{array}{l}\text { POTS (deconditioning and } \\
\text { hyperadrenergic) }\end{array}$ \\
\hline 2 & $\mathrm{~F}$ & 33 & 0 & $\begin{array}{l}\text { Fatigue, SOB, OI, LH, syncope, } \\
\text { anosmia, dysgeusia }\end{array}$ & LH, syncope, early satiety, WL & POTS (hypovolemic) \\
\hline 3 & M & 21 & 66 & HA, anosmia, insomnia & LH & POTS (hyperadrenergic) \\
\hline 4 & $\mathrm{~F}$ & 34 & 0 & $\begin{array}{l}\text { Palpitations, flushing, OI, fatigue, } \\
\text { hypohidrosis, heat intolerance, } \\
\text { urinary hesitancy }\end{array}$ & $\begin{array}{l}\text { LH, flushing, hypohidrosis, heat } \\
\text { intolerance, urinary hesitancy }\end{array}$ & AAG \\
\hline 5 & $\mathrm{~F}$ & 72 & 0 & $\begin{array}{l}\text { HA, myalgia, insomnia, anorexia, } \\
\text { SOB, cough, fatigue, LH, brain } \\
\text { fog }\end{array}$ & LH & OI \\
\hline 6 & $\mathrm{~F}$ & 42 & 0 & Anosmia, dysgeusia, LH & LH & OI \\
\hline 7 & $\mathrm{~F}$ & 29 & 79 & $\begin{array}{l}\text { Fatigue, worsening Sx of ulcera- } \\
\text { tive colitis (diarrhea) }\end{array}$ & LH, flushing & POTS (deconditioning) \\
\hline 8 & M & 37 & 20 & SOB, fatigue, $\mathrm{CP}$, cough & LH & Vasodepressor syncope \\
\hline 9 & $\mathrm{~F}$ & 60 & 2 & Fever, cough, HA, fatigue & LH, orthostatic HA & OI \\
\hline 10 & M & 77 & 0 & $\begin{array}{l}\text { Generalized weakness, LH, } \\
\text { tremor, SOB anorexia, WL, } \\
\text { cough }\end{array}$ & LH & $\mathrm{LBD}$, exacerbation of $\mathrm{nOH}$ \\
\hline 11 & $\mathrm{~F}$ & 67 & 0 & $\begin{array}{l}\text { Vision change, foot pain, paresthe- } \\
\text { sia, hyperhidrosis, HA, fatigue, } \\
\text { LH }\end{array}$ & Burning pain, LH & $\begin{array}{l}\text { Exacerbation of sensory-predom- } \\
\text { inant autonomic neuropathy }\end{array}$ \\
\hline 12 & M & 39 & 101 & $\begin{array}{l}\text { Anosmia, dysgeusia, flu-like } \mathrm{Sx} \text {, } \\
\text { fatigue, cough, diarrhea }\end{array}$ & Syncope (cough-associated) & $\begin{array}{l}\text { Cough-associated syncope } \\
\text { (vasovagal) }\end{array}$ \\
\hline 13 & M & 36 & 31 & SOB, DVT, tremulousness & Syncope, LH, numbness & $\begin{array}{l}\text { Inappropriate sinus tachycardia, } \\
\text { SFN exacerbation }\end{array}$ \\
\hline 14 & $\mathrm{~F}$ & 60 & 10 & $\mathrm{SOB}, \mathrm{CP}$ & LH & OI \\
\hline 15 & $\mathrm{~F}$ & 35 & 75 & $\begin{array}{l}\text { Diarrhea, nausea, SOB, CP, lym- } \\
\text { phadenopathy }\end{array}$ & LH, burning pain, sicca symptoms & POTS (neuropathic) \\
\hline 16 & $\mathrm{~F}$ & 56 & 27 & Sore throat, fever, anosmia & LH, orthostatic HA, hyperhidrosis & OI \\
\hline 17 & $\mathrm{~F}$ & 59 & 32 & $\begin{array}{l}\text { Cough, SOB, fever, nausea, diar- } \\
\text { rhea }\end{array}$ & $\mathrm{LH}$ & OI \\
\hline 18 & M & 40 & 3 & $\begin{array}{l}\text { Myalgia, brain fog, WL, general- } \\
\text { ized weakness, HA }\end{array}$ & $\begin{array}{l}\text { Orthostatic HA, WL, LH, hyper- } \\
\text { hidrosis }\end{array}$ & POTS (hyperadrenergic) \\
\hline 19 & $\mathrm{~F}$ & 37 & 0 & $\begin{array}{l}\text { Fatigue, insomnia, fog, thirst, LH, } \\
\text { urinary urgency }\end{array}$ & $\mathrm{LH}$, urinary urgency & OI \\
\hline 20 & M & 33 & 0 & SOB, fatigue, $\mathrm{LH}$ & LH & OI \\
\hline 21 & $\mathrm{~F}$ & 48 & 0 & $\mathrm{HA}$, fatigue, $\mathrm{LH}$ & Orthostatic HA, LH & OI \\
\hline 22 & $\mathrm{~F}$ & 45 & 0 & $\begin{array}{l}\text { Myalgia, HA, SOB, fatigue, palpi- } \\
\text { tations, LH }\end{array}$ & $\begin{array}{l}\text { LH, orthostatic HA and tachy- } \\
\text { cardia }\end{array}$ & OI \\
\hline 23 & M & 52 & 21 & Fatigue, $\mathrm{SOB}, \mathrm{CP}$ & Burning pain, allodynia & SFN exacerbation \\
\hline 24 & M & 38 & 0 & $\begin{array}{l}\text { Fever, myalgia, SOB, pain, } \mathrm{LH} \text {, } \\
\text { ear ringing, blurry vision, brain } \\
\text { fog, anxiety }\end{array}$ & $\mathrm{LH}$, orthostatic blurry vision & OI \\
\hline 25 & M & 49 & 15 & Fever, cough, chills & LH & OI \\
\hline 26 & M & 54 & 7 & Fatigue, fever, cough, SOB, CP & LH, orthostatic tachycardia & OI \\
\hline 27 & $\mathrm{~F}$ & 27 & 7 & HA, fatigue, SOB, panic & $\mathrm{LH}$, orthostatic HA & OI \\
\hline
\end{tabular}

Pt patient, Sx symptoms, LH lightheadedness, POTS postural orthostatic tachycardia syndrome, SOB shortness of breath, OI orthostatic intolerance, $H A$ orthostatic headache, $A A G$ autoimmune autonomic ganglionopathy, $n O H$ neurogenic orthostatic hypotension, $L B D$ Lewy body dementia, $D V T$ deep vein thrombosis, $C P$ chest pain, $W L$ weight loss, $S F N$ small fiber neuropathy 


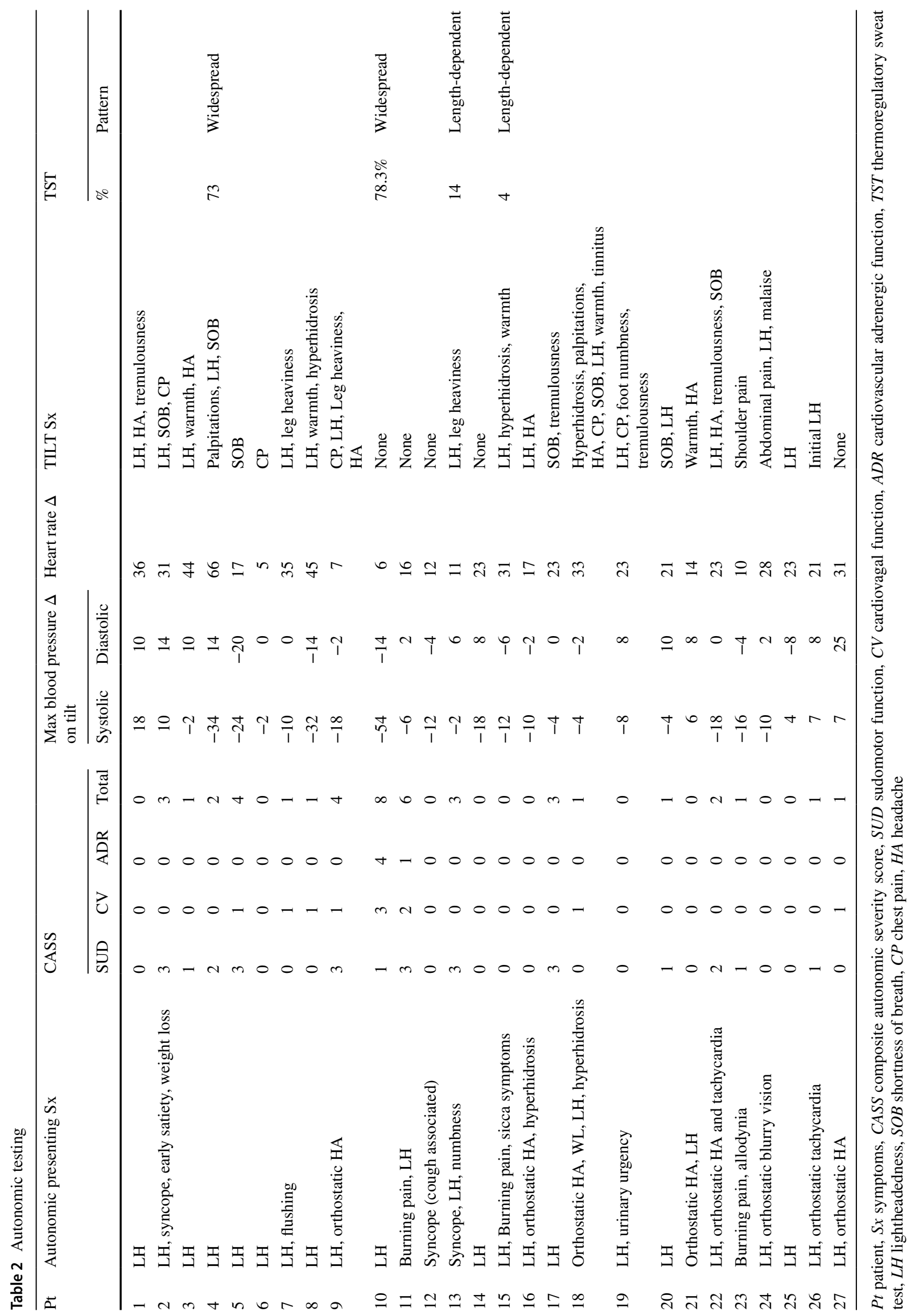




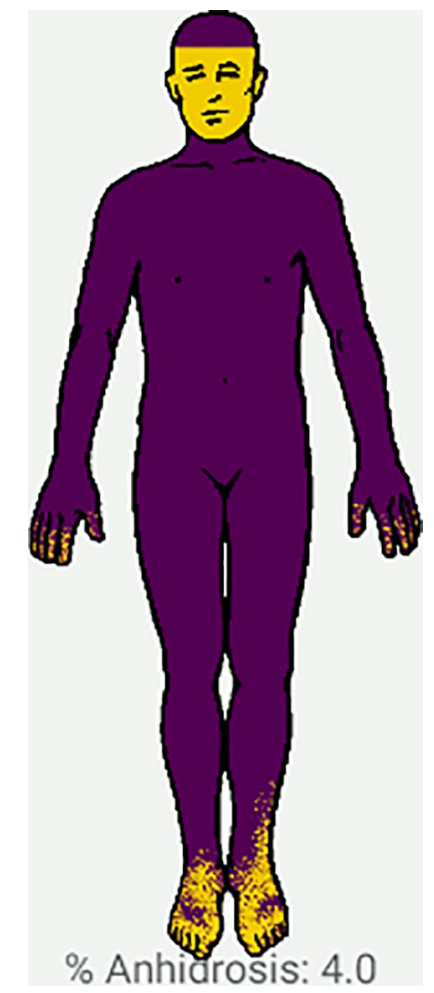

Fig. 1 Thermoregulatory sweat test using alizarin red powder showing length-dependent anhidrosis of the fingers, left lower leg, and bilateral feet. The findings are consistent with length-dependent autonomic (small fiber) neuropathy. Hidrotic areas are colored in purple and anhidrotic areas in yellow; the face was not powdered

warmth) and length-dependent anhidrosis of the feet and fingertips (Fig. 1).

This case represents neuropathic POTS. The etiology is likely a postinfectious form of a limited autonomic (small fiber) neuropathy.

Patient 1 A 35-year-old woman with obesity and anxiety contracted SARS-CoV-2 with multiple symptoms including fatigue, chest pain, and tachycardia. She quarantined with a limited level of activity until symptoms improved, and returned to work after several weeks. At that time, she was in contact with a co-worker who tested positive for COVID19 and had to quarantine again. While she did not get reinfected, she continued to have fatigue, brain fog, chest pain, insomnia, and decreased level of activity. She presented about 4 months after the initial infection with orthostatic lightheadedness, tachycardia, and, later, hyperhidrosis. Examination was normal except for signs of deconditioning. Her chest pain was diagnosed as costochondritis.

Autonomic reflex screen revealed excessive heart rate acceleration during tilt meeting POTS criteria, with hyperadrenergic characteristics including an increase in blood pressure on tilt and resting sweat activity on QSART.
This case represents POTS with features of both deconditioning and a hyperadrenergic state. The patient was treated with a rehabilitative reconditioning program, cognitive behavioral therapy, and low-dose beta-blocker with subsequent improvement and was able to return to work part-time after 3 months.

\section{Mild orthostatic intolerance}

Patient 24 A 38-year-old man developed dyspnea, fever, and myalgia. He tested positive for SARS-CoV-2. Concomitant with his diagnosis, he experienced fragmented sleep, postural lightheadedness, blurred vision, tinnitus, brain fog, visceral discomfort, and anxiety. He was worried that he would not wake up the next day and would leave behind his young son. This led to multiple emergency department visits. He presented a month after his diagnosis with worsening lightheadedness and fatigue that prevented him from working or exercising. Extensive testing for his visceral discomfort revealed no organic etiology. His neurological examination was normal. ARS revealed normal autonomic reflexes, low-normal baseline blood pressure, and a generous heart rate increase $(28 \mathrm{bpm})$ on tilt table testing associated with lightheadedness.

The case signifies that orthostatic symptoms can happen para-infectiously and be perpetuated by disease anxiety and deconditioning that outlive the acute phase of the infection. Treatment included reassurance, anxiolytics, and a rehabilitative reconditioning program. However, the patient continued to seek evaluation for his visceral pain and remained symptomatic 2 months after evaluation.

\section{Small fiber/autonomic neuropathy}

Patient 23 A 52-year-old man previously of excellent health contracted SARS-CoV-2. He experienced moderate respiratory symptoms of shortness of breath and productive cough and was managed in the outpatient setting. About 3 weeks later, he began to experience burning pain in the feet that spread up to the knees that was associated with imbalance and falls. The pain would wake him at night, impacted his functional capacity, and was associated with allodynia. Neurological examination revealed high arches, moderate distal sensory loss to pinprick, and antalgic gait, but was otherwise normal including all large fiber sensory modalities. ARS was normal apart from a relatively reduced QSART response at the foot compared to proximal responses, compatible with distal small fiber neuropathy (Fig. 2). Gabapentin and a topical lidocaine cream improved his neuropathic symptoms.

The patient's presentation is most compatible with a small fiber-predominant sensory neuropathy unmasked by COVID-19 infection. The high arches suggest an 


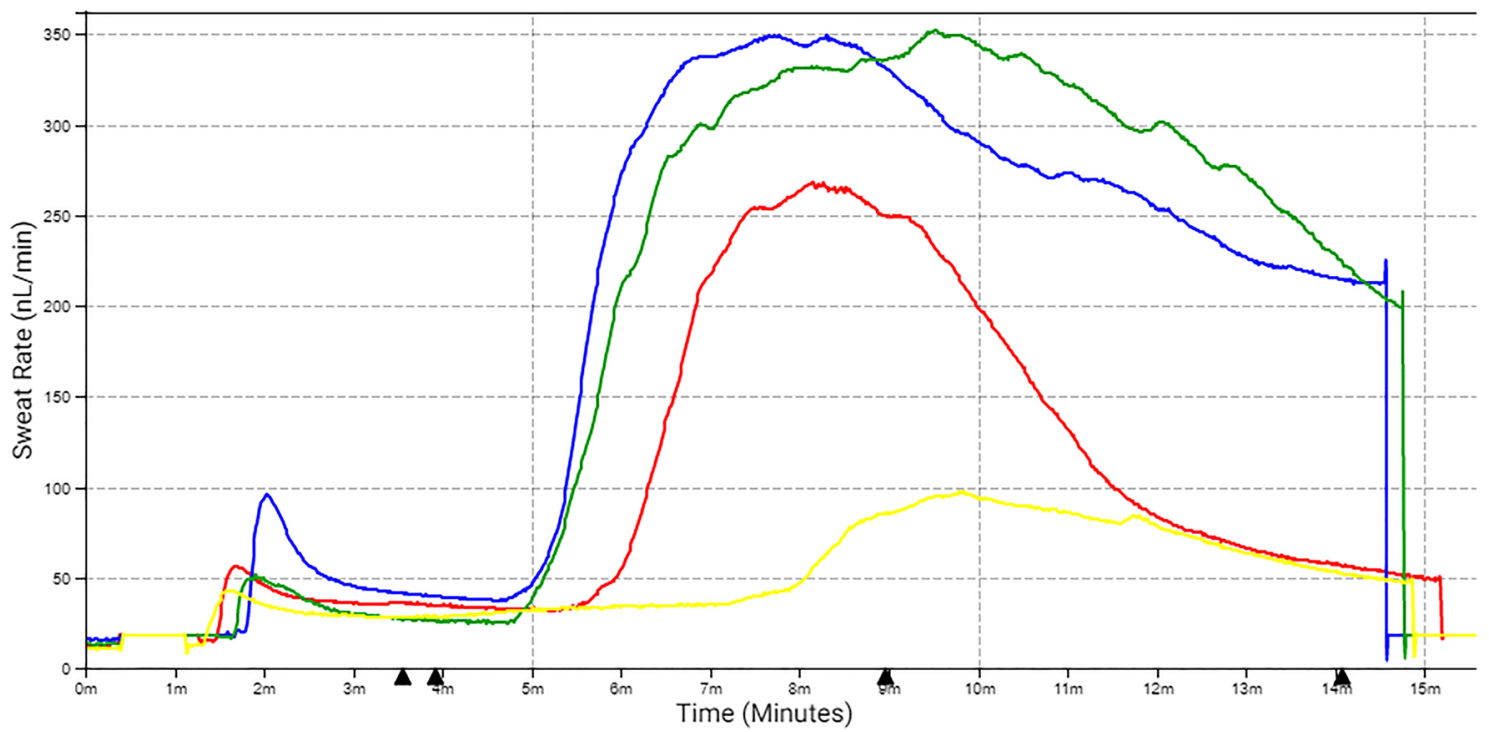

Fig. 2 Quantitative sudomotor axon reflex test showing relative distal (foot site) anhidrosis indicating distal autonomic (small fiber) neuropathy. Sweat output $(\mu \mathrm{L})$ : forearm site (red) 1.03 (5th percen-

otherwise - until the infection-asymptomatic hereditary/ genetic form of neuropathy.

Patient 11 A 67-year-old woman presented with longstanding biopsy-proven length-dependent small fiber neuropathy in the setting of psoriatic arthritis. Her symptoms of mild acral tingling and burning pain had been stable for 10 years, until she contracted SARS-CoV-2. Concomitant with her viral illness, she developed severe burning pain in the hands and feet. She presented 6 months later with persistent symptoms and occasional orthostasis. Her neurological examination revealed hyperalgesia in the hands and feet. ARS revealed patchy loss of QSART responses in the lower extremity, mild to moderate cardiovagal dysfunction, and mild adrenergic impairment. The patient's pain management was optimized, and she was started on a rehabilitative program.

This is an example of a chronic premorbid sensory and small fiber-predominant autonomic neuropathy exacerbated by COVID-19 infection.

\section{Autoimmune autonomic ganglionopathy}

Patient 4 A 33-year-old right-handed woman with a history of episodes of labile, paroxysmal hypertension and hypotension in the setting of stressful events (including pregnancy and delivery), mild heat intolerance, early satiety, and chronic constipation abruptly developed lightheadedness, facial flushing, shaking, prominent labile hypertension and hypotension, and labile heart rate, tinnitus, vision changes, brain fog, and heat intolerance with frank anhidrosis. Addi- tile $=0.25)$, proximal leg (blue) 2.11 (5th percentile $=0.48)$, distal leg (green) 2.30 (5th percentile $=0.62$ ), and foot (yellow) 0.26 (5th percentile $=0.15$ and 10 th percentile $=0.33$ )

tionally, the patient experienced worsening early satiety but improvement of her chronic constipation. She unintentionally lost 10 pounds. She had no symptoms of upper respiratory infection or gastroenteritis and hence did not have COVID-19 PCR testing. However, since other family members had tested positive for COVID-19, she underwent antibody testing a month and a half after symptom onset and was positive for immunoglobulin (Ig) $\mathrm{M}$ but negative for IgG COVID-19 antibodies.

The patient was assessed locally, suspected of having POTS, and started on propranolol. She also had a paraneoplastic autoantibody evaluation, and her ganglionic acetylcholine receptor antibody was $111 \mathrm{pmol} / \mathrm{L}$ [normal $<53 \mathrm{pmol} / \mathrm{L}$ ], which prompted referral to our institution.

She presented to us 5 months after symptom onset. Neurological examination was normal. Her ARS showed prominently labile blood pressure (meeting criteria for orthostatic hypotension) and tachycardia (Fig. 3a), and her TST revealed patchy widespread anhidrosis of $73 \%$ (Fig. 3b). Given her presentation, pattern of anhidrosis, and history of antibody positivity, the patient was diagnosed with a forme fruste of AAG and started on intravenous methylprednisolone. She had initial improvement followed by a relapse in symptoms after a COVID-19 reinfection.

The case likely represents a forme fruste of autonomic ganglionopathy. The history of similar, fluctuating symptoms in the past may indicate a chronic form that was exacerbated by COVID-19 infection. The autoantibody positivity and improvement with immunotherapy support a primary autoimmune etiology. 

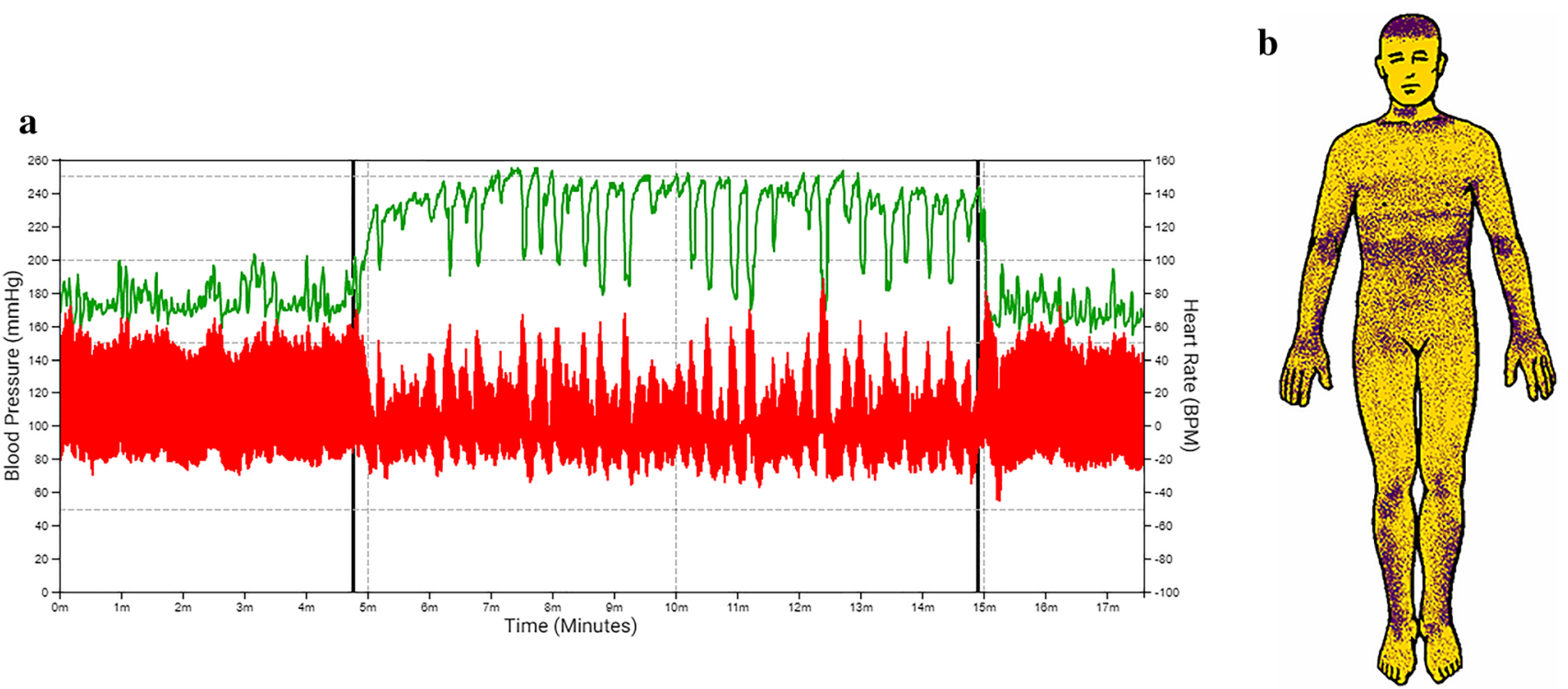

Fig. 3 a Hemodynamic profile on head-up tilt study. Note prominent orthostatic tachycardia 129-42 bpm (green) and labile orthostatic hypotension (red) with systolic blood pressure change ranging from

For all the cases above, alternative etiologies of autonomic dysfunction were investigated and were absent or insufficient to explain the patients' complaints.

\section{Discussion}

In this study, we report the largest cohort to date of patients with symptoms concerning for autonomic dysfunction arising in the para-infectious or postinfectious period of COVID-19. All the patients had thorough, validated autonomic testing and careful clinical assessments to explore the association of often vague, nonspecific postinfectious symptoms with objective abnormalities on autonomic function testing. Remarkably, 17/27 (63\%) patients had abnormalities on autonomic function testing, ranging from symptomatic orthostatic tachycardia to severe autonomic failure. The most common autonomic presentation post-COVID-19 was OI. While some of these patients fulfilled criteria for POTS or had milder changes in orthostatic heart rate and blood pressure providing objective signs of orthostatic intolerance, the majority of patients experiencing orthostatic symptoms had a normal tilt study.

Schondorf and Low first described postural orthostatic tachycardia syndrome in 1993 [20]. Seven out of the 16 reported patients in this landmark study were classified as post-viral, as they had symptoms of viral upper respiratory tract infection prior to the onset of their symptoms. It was proposed that these patients experience a form of postinfectious limited autonomic neuropathy. Similarly, other types
-14 to $-34 \mathrm{mmHg}$. b Thermoregulatory sweat test showing widespread anhidrosis (73\%) in a patchy pattern compatible with autonomic ganglionopathy

of autonomic neuropathy have been reported after a variety of infections [21, 22]. Despite the limited experience, there is evidence that patients with existing neurological disease may develop worsening of their neurological symptoms with COVID-19 and have a more severe course [23].

Patients infected with SARS-CoV-2 can develop a wide range of clinical manifestations including fatigue, myalgias, and gastrointestinal dysfunction [24-26]. These in turn may lead to deconditioning, weight loss, and hypovolemia, which are known to predispose to orthostatic intolerance. It would therefore be expected that patients with a protracted course of COVID-19 infection or with lingering postinfectious symptoms are at increased risk of developing orthostatic intolerance related to those factors. Furthermore, healthrelated anxiety and insomnia in relation to COVID-19 itself or disease-related measures such as quarantining [27] are likely risk factors for worsening or emergence of a hyperadrenergic state and deconditioning, further increasing the risk of resulting autonomic symptoms.

As expected, many patients with OI had clinical features consistent with deconditioning, hypovolemia, and/or a hyperadrenergic state. Although the methodology used in our report does not allow an accurate measure of the frequency of OI following COVID-19 infection, symptoms compatible with OI are anecdotally commonly encountered in Mayo Clinic's post-COVID clinic, which may suggest that postinfectious OI is more common following COVID19 compared to other viral infections. The limited currently available data do not suggest that OI following COVID-19 differs from that encountered following other viral disorders, 
but further research into this phenomenon is needed to solidify this impression. Symptoms induced by the upright position without a clear cardiovascular correlate is not unique to this cohort, but the high percentage of patients in this category may indicate an increased level of anxiety, overlap with chronic fatigue and central sensitization, or a condition in the category of persistent postural perceptual dizziness.

We also identified patients with autonomic and small fiber neuropathies, which may not be surprising considering that postinfectious neuropathy has been reported after a variety of viral infections such as herpes simplex and infectious mononucleosis [21, 22]. Interestingly, while one of our patients developed de novo limited autonomic and small fiber neuropathy with POTS following COVID-19 infection, other cases were characterized by postinfectious worsening of preexisting autonomic/small fiber neuropathies. That was also true for the patient with presumed preexisting AAG. While a causative relationship between these neuropathies or their exacerbation and the preceding COVID-19 infection cannot be proven, the temporal association and the frequency within this relatively small cohort is intriguing. The mechanisms underlying this phenomenon are unclear at present and deserve further study. Exacerbation of a single case of neurogenic orthostatic hypotension due to a synucleinopathy following COVID-19 infection could be coincidental, but this observation also deserves further surveillance and study.

Our study has several limitations. In addition to referral bias, the retrospective design is subject to recall bias, and bias is also introduced due to reliance on documentation in the medical record and missing data. As the study is purely observational, we can only report associations and cannot draw reliable conclusions regarding causative relationships or underlying mechanisms.

We conclude that many patients referred for autonomic function testing after experiencing prolonged symptoms after COVID-19 infection have abnormalities on autonomic function testing that are usually mild. Orthostatic symptoms are commonly encountered and in some are associated with objective findings of POTS or milder degrees of OI, although most commonly these symptoms are not associated with objective findings during head-up tilt. In both scenarios, symptoms may linger for a prolonged period and limit activities of daily living to varying degrees. Post-COVID autonomic and small fiber neuropathy are encountered as well, often as an exacerbation of preexisting conditions.

Expanded retrospective and prospective studies are needed to further explore and characterize the spectrum of autonomic dysfunction related to COVID-19 infection, understand its natural history, and optimize its management.

Acknowledgements This work was supported in part by the National Institutes of Health (NS 926250), Mayo Clinic CTSA (UL1 TR000135), and Mayo Clinic Funds.

\section{Declarations}

Conflict of interest On behalf of all authors, the corresponding author states that there is no conflict of interest.

\section{References}

1. Centers for Disease Control and Prevention (2021) COVID-19 Cases, data and surveillance. URL https://www.cdc.gov/coron avirus/2019-ncov/cases-updates/ (Accessed 27 Feb 2021).

2. Koralnik IJ, Tyler KL (2020) COVID-19: a global threat to the nervous system. Ann Neurol 88:1-11. https://doi.org/10.1002/ ana. 25807

3. Harapan BN, Yoo HJ (2021) Neurological symptoms, manifestations, and complications associated with severe acute respiratory syndrome coronavirus 2 (SARS-CoV-2) and coronavirus disease 19 (COVID-19). J Neurol 23:1-13

4. Collins FS (2021) NIH launches new initiative to study "Long COVID". URL: https://www.nih.gov/about-nih/who-we-are/ nih-director/statements/nih-launches-new-initiative-study-longcovid. Accessed 27 Feb 2021

5. Miglis MG, Prieto T, Shaik R, Muppidi S, Sinn D-I, Jaradeh S (2020) A case report of postural tachycardia syndrome after COVID-19. Clin Auton Res 30:449-451. https://doi.org/10. 1007/s10286-020-00727-9

6. Kanjwal K, Jamal S, Kichloo A, Grubb BP (2020) New-onset postural orthostatic tachycardia syndrome following coronavirus disease 2019 infection. J Innov Card Rhythm Manag 11:4302-4304. https://doi.org/10.19102/icrm.2020.111102

7. Umapathi T, Poh MQW, Fan BE, Li KFC, George J, Tan JYL (2020) Acute hyperhidrosis and postural tachycardia in a COVID-19 patient. Clin Auton Res 30:571-573. https://doi. org/10.1007/s10286-020-00733-x

8. Novak P (2020) Post COVID-19 syndrome associated with orthostatic cerebral hypoperfusion syndrome, small fiber neuropathy and benefit of immunotherapy: a case report. Neurological Sci. https://doi.org/10.1016/j.ensci.2020.100276

9. Lamotte G, Benarroch EE, Coon EA (2021) Paroxysmal hypothermia and hyperhidrosis with exacerbation after COVID-19 Infection. Clin Auton Res. https://doi.org/10.1007/s10286-02100783-9 (Epub ahead of print)

10. Mostel Z, Ayat P, Capric V, Trimmingham A, McFarlane SI (2021) Guillain-Barré syndrome in a COVID-19 patient: a case report and review of management strategies. Am J Med Case Rep 9:198-200. https://doi.org/10.12691/ajmcr-9-3-16

11. Türk Börü Ü, Köseoğlu Toksoy C, Bölük C, Demirbaş H, Yılmaz AÇ (2021) A case of Guillain-Barré syndrome related to COVID-19 infection. Int J Neurosci 12:1-3. https://doi.org/ 10.1080/00207454.2021.1886097

12. Goldstein DS, Cheshire WP Jr (2017) The autonomic medical history. Clin Auton Res 27:223-233

13. Cheshire WP, Freeman R, Gibbons CH, Cortelli P, Wenning GK, Hilz MJ, Spies JM, Lipp A, Sandroni P, Wada N, Mano A, Ah Kim H, Kimpinski K, Iodice V, Idiáquez J, Thaisetthawatkul P, Coon EA, Low PA, Singer W (2021) Electrodiagnostic assessment of the autonomic nervous system: a consensus statement endorsed by the American Autonomic Society, American Academy of Neurology, and the International Federation of Clinical Neurophysiology. Clin Neurophysiol 132:666-682

14. Low PA (1993) Autonomic nervous system function. J Clin Neurophysiol 10:14-27 
15. Low PA, Caskey PE, Tuck RR, Fealey RD, Dyck PJ (1983) Quantitative sudomotor axon reflex test in normal and neuropathic subjects. Ann Neurol 14:573-580

16. Pickering TG, Sleight $P$ (1969) Quantitative index of baroreflex activity in normal and hypertensive subjects using Valsalva's manoeuvre. Br Heart J 31:392

17. Vogel ER, Sandroni P, Low PA (2005) Blood pressure recovery from Valsalva maneuver in patients with autonomic failure. Neurology 65:1533-1537

18. Low PA (1993) Composite autonomic scoring scale for laboratory quantification of generalized autonomic failure. Mayo Clin Proc 68:748-752. https://doi.org/10.1016/s0025-6196(12)60631-4

19. Fealey RD, Low PA, Thomas JE (1989) Thermoregulatory sweating abnormalities in diabetes mellitus. Mayo Clin Proc 64:617-628

20. Schondorf R, Low PA (1993) Idiopathic postural orthostatic tachycardia syndrome: an attenuated form of acute pandysautonomia? Neurology 43:132-137. https://doi.org/10.1212/wnl.43.1_part_1. 132

21. Neville BG, Sladen GE (1984) Acute autonomic neuropathy following primary herpes simplex infection. J Neurol Neurosurg Psychiatry 47:648-650. https://doi.org/10.1136/jnnp.47.6.648

22. Vassallo M, Camilleri M, Caron BL, Low PA (1991) Gastrointestinal motor dysfunction in acquired selective cholinergic dysautonomia associated with infectious mononucleosis. Gastroenterology 100:252-258. https://doi.org/10.1016/0016-5085(91) 90609-o

23. Kubota T, Kuroda N (2021) Exacerbation of neurological symptoms and COVID-19 severity in patients with preexisting neurological disorders and COVID-19: a systematic review. Clin Neurol Neurosurg 200:106349. https://doi.org/10.1016/j.clineuro.2020. 106349

24. Zhu N, Zhang D, Wang W et al (2020) A novel coronavirus from patients with pneumonia in China, 2019. N Engl J Med 382:727733. https://doi.org/10.1056/NEJMoa2001017

25. Chan JF, Yuan S, Kok KH et al (2020) A familial cluster of pneumonia associated with the 2019 novel coronavirus indicating person-to-person transmission: a study of a family cluster. Lancet 395:514-523. https://doi.org/10.1016/S0140-6736(20)30154-9

26. Huang C, Wang Y, Li X et al (2020) Clinical features of patients infected with 2019 novel coronavirus in Wuhan, China. Lancet 395:497-506

27. Wang C, Song W, Hu X, Yan S, Zhang X, Wang X, Chen W (2021) Depressive, anxiety, and insomnia symptoms between population in quarantine and general population during the COVID19 pandemic: a case-controlled study. BMC Psychiatry 21:99. https://doi.org/10.1186/s12888-021-03108-2 